\title{
On the Laplacian and signless Laplacian polynomials of graphs with semiregular automorphisms
}

\author{
Majid Arezoomand ${ }^{1}$
}

Received: 20 November 2017 / Accepted: 25 May 2019 / Published online: 4 June 2019

(c) Springer Science+Business Media, LLC, part of Springer Nature 2019

\begin{abstract}
A graph $\Gamma$ is called $n$-Cayley graph over a group $G$ if $\operatorname{Aut}(\Gamma)$ has a semiregular subgroup isomorphic to $G$ with $n$ orbits (of equal size). In this paper, we give a decomposition of the Laplacian and signless Laplacian polynomials of $n$-Cayley graphs in terms of irreducible representations of $G$. Also, we construct several families of graphs with integral Laplacian and signless Laplacian spectrum.
\end{abstract}

Keywords n-Cayley graph · Laplacian eigenvalue - Signless Laplacian eigenvalue · Integral graph

Mathematics Subject Classification 05C50 - 05C25 - 05C31

\section{Introduction}

In this paper, a graph means a finite, undirected and simple graph and a group is finite unless specified otherwise. For a graph $\Gamma$, we use $V(\Gamma), E(\Gamma)$ and $\operatorname{Aut}(\Gamma)$ to denote its vertex set, edge set and automorphism group. For the most part, our notation and terminology are standard and mainly taken from [5] (for graph theory) and [15] (for representation theory of finite groups). For the graph-theoretic and group-theoretic terminology not defined here, we refer the reader to $[5,15]$.

A graph $\Gamma$ is called an $n$-Cayley graph over a group $G$ if there exists a semiregular subgroup of $\operatorname{Aut}(\Gamma)$ isomorphic to $G$ with $n$ orbits (of equal size). A graph is called a Cayley graph over a group $G$ if its automorphism group contains a regular subgroup isomorphic to $G$. Indeed, every Cayley graphs is a 1-Cayley graph. Also, a Cayley graph over a finite group $G$ having a subgroup $H$ of index $n$ is an $n$-Cayley graph over $H$ [1, Lemma 8]. Also, $n$-Cayley graphs over cyclic groups are called $n$-circulant. In particular, 2-Cayley and 3-Cayley graphs over cyclic groups are called bicirculant

\footnotetext{
Majid Arezoomand

arezoomand@lar.ac.ir

1 University of Larestan, Larestan 74317-16137, Iran
} 
and tricirculant graphs [23], respectively. Unlike Cayley graphs, in general $n$-Cayley graphs are not vertex transitive for $n \geq 2$. Furthermore, there are vertex-transitive $n$-Cayley graphs which are not Cayley graphs such as generalized Petersen graphs.

Undirected and loop-free 2-Cayley graphs are by some authors called semi-Cayley graphs [2,27] and also bi-Cayley graphs [18]. In this paper, we follow [27] to use the term semi-Cayley.

$n$-Cayley graphs, in particular when $n=2$ or $n=3$, have been playing an important role in many classical fields of graph theory, such as strongly regular graphs [19, $20,22,23,27]$, symmetry properties of graphs $[7,8,21]$ and the spectrum of graphs $[1,3,4,11,12]$.

The following characterization of (undirected and without loop) $n$-Cayley graphs is given by the present author et. al in [1, Lemma 2]. Let $T_{i j}, 1 \leq i, j \leq n$, be $n^{2}$ subsets of a group $G$ such that $T_{i j}^{-1}=T_{j i}$ (here $X^{-1}=\left\{x^{-1} \mid x \in X\right\}$ ) and $1 \notin T_{i i}$ for all $1 \leq i, j \leq n$. Define a graph $\Gamma$ with vertex set $G \times\{1, \ldots, n\}$ and with vertices $(g, i),(h, j)$ adjacent if and only if $h g^{-1} \in T_{i j}$. Then, $\Gamma$ is an $n$-Cayley graph over $G$, and conversely, every $n$-Cayley graph over $G$ can be obtain in this way. In particular, let $\Gamma$ be a semi-Cayley graph over a group $G$. Then, there exists subsets $T_{11}=R=R^{-1}, T_{22}=S=S^{-1}, 1 \notin R \cup L, T_{12}=T$ and $T_{21}=T^{-1}$ of $G$ such that $\Gamma=\operatorname{Cay}\left(G ; T_{i j} \mid 1 \leq i, j \leq 2\right)$. Thus, the presentation of $\Gamma$ only depends on $R, S$ and $T$. So we denote $\Gamma$ by $\operatorname{SC}(G ; R, S, T)$.

Let $\Gamma$ be a graph with vertex set $\left\{v_{1}, \ldots, v_{n}\right\}$. The adjacency matrix of $\Gamma$ is an $n \times n$ matrix $A=\left[a_{i j}\right]$, where $a_{i j}=1$ whenever $v_{i}$ and $v_{j}$ are adjacent and $a_{i j}=0$, otherwise. The degree matrix of $\Gamma$ is a diagonal $n \times n$ matrix $D=\operatorname{diag}\left(d_{1}, \ldots, d_{n}\right)$, where $d_{i}$ is the number of vertices adjacent to $v_{i}$. The matrices $L=D-A$ and $Q=$ $D+A$ are called Laplacian and signless Laplacian matrices of $\Gamma$, respectively. The characteristic polynomial of an $n \times n$ matrix $X$ is $\chi_{X}(\lambda)=\operatorname{det}\left(\lambda I_{n}-X\right)$, where $I_{n}$ is the $n \times n$ identity matrix and the roots of this polynomial are called eigenvalues of $X$. In this paper, the eigenvalues, Laplacian eigenvalues and signless Laplacian eigenvalues of a graph $\Gamma$ are eigenvalues of adjacency, Laplacian and signless Laplacian matrices of $\Gamma$, respectively.

It is known that numerous proofs in graph theory depend on the spectrum (the multiset of eigenvalues) of graphs and the spectrum of a graph is one of the most important algebraic invariants. The basic relationships between algebraic properties of these eigenvalues and the usual properties of graphs are available in [5]. Also, Laplacian eigenvalues of a graph can be used in several areas of mathematical research and have a physical interpretation in various physical and chemical theories. For a survey of known results about the spectrum of Laplacian matrices of graphs until 1991, we refer the reader to [26]. The adjacency and Laplacian matrix of a graph were much more investigated in the past than the signless Laplacian matrix. Since 2005, several papers on the signless Laplacian eigenvalues of a graph have been published. For a survey on this topic, we refer to [6].

There are several papers relating to the spectrum of Cayley graphs. Since Cayley graphs are regular, one can obtain their Laplacian and signless Laplacian from their adjacency spectrum. But $n$-Cayley graphs are not necessarily regular. So it seems that computing their Laplacian and signless Laplacian eigenvalues is important. Spectrum 
of semi-Cayley graphs over abelian groups are computed in 2010 [11]. This result was improved for $n$-Cayley graphs over arbitrary groups in 2013 by the present author and Taeri [1]. In 2015, the Laplacian and signless Laplacian spectrum of semi-Cayley graphs over abelian groups is computed by Gao et al [12]. It seems that their argument cannot be extended to non-abelian groups or $n$-Cayley graphs for $n \geq 3$. In this paper, we improve this result. We find a factorization of the characteristic polynomial of $M_{\alpha}(\Gamma):=D+\alpha A$, where $D$ and $A$ are the degree and adjacency matrices of an $n$-Cayley graph $\Gamma$ over a group $G$ and $\alpha$ is a number, in terms of irreducible representations of $G$. In particular, we compute the Laplacian and signless Laplacian eigenvalues of semi-Cayley graphs and tri-Cayley graphs over abelian groups. Also, we compute the Laplacian and signless Laplacian eigenvalues of $n$-sunlet graph and Tutte's 8-cage graph as a result of our main result.

One of the themes that has arisen in the literature on Laplacian (signless Laplacian) eigenvalues for graphs is that of Laplacian (signless Laplacian) integral graphs-i.e., those graphs whose all of their Laplacian (signless Laplacian) eigenvalues are integers. In $[9,13,14,24,25,29]$, various families of Laplacian and signless Laplacian integral graphs were identified. Also, [16,17,28,30] provide constructions for certain classes of Laplacian and signless Laplacian integral graphs. It is not difficult to see that any regular graph that is adjacency integral (i.e., all eigenvalues of its adjacency matrix are integers) is necessarily Laplacian and signless Laplacian integral as well. One of the interesting challenges in dealing with Laplacian (signless Laplacian) integral graphs is that of describing, constructing and understanding families of Laplacian (signless Laplacian) integral graphs that are not regular. In this paper, we construct several Laplacian and signless Laplacian integral $n$-Cayley graphs.

\section{Main results}

The author et al. determined the characteristic polynomial of an $n$-Cayley graph over finite group $G$ in [1, Theorem 6]. In this paper, we compute the Laplacian and signless Laplacian polynomial of $n$-Cayley graphs. First, let us recall some notations of [1]. Let $G$ be a finite group and $\mathbb{C}[G]$ be the $\mathbb{C}$-vector space of all complex-valued functions on $G$ with basis $\left\{e_{g} \mid g \in G\right\}$, where

$$
e_{g}(h)= \begin{cases}1 & h=g \\ 0 & h \neq g\end{cases}
$$

Let $\operatorname{Irr}(G)=\left\{\varrho^{(1)}, \ldots, \varrho^{(m)}\right\}$ be the set of all irreducible inequivalent complex matrix representations of $G$ and $d_{k}$ be the degree of $\varrho_{k}, k=1, \ldots, m$. Let $\varrho_{i j}^{(k)}(g)$ be the $i j$ th entry of $\varrho^{(k)}(g), 1 \leq i, j \leq d_{k}$ and $\bar{\varrho}_{i j}^{(k)}=\sum_{g \in G} \overline{\varrho_{i j}^{(k)}(g)} e_{g}$, where $\bar{z}$ denotes the complex conjugate of the complex number $z$. For a $1 \times m$ vector $v$ and $1 \leq i \leq n$, we define $v^{i}$ to be a $1 \times n m$ vector with $n$ blocks, whose $i$ th block is $v$ and other blocks are $0_{1 \times m}$. So $e_{g}^{i}$ is the $1 \times n|G|$ vector with $n$ blocks, where $i$ th block is $e_{g}$, as defined, and other blocks are $0_{1 \times|G|}$ vectors. Let $V$ be the vector space with basis $\left\{e_{g}^{i} \mid g \in G, 1 \leq i \leq n\right\}, \operatorname{dim}_{C}(V)=n|G|$, and we may view the adjacency 
matrix $A$ of an $n$-Cayley graph $\Gamma=\operatorname{Cay}\left(G ; T_{i j} \mid 1 \leq i, j \leq n\right)$ of $G$ as a linear map $A: V \rightarrow V$. Keeping these notations, we have

Theorem 1 (1) $\mathscr{B}=\left\{\bar{\varrho}_{i j}^{(l), k} \mid 1 \leq i, j \leq d_{l}, 1 \leq l \leq m, 1 \leq k \leq n\right\}$ is a basis for $V$, where $\bar{\varrho}_{i j}^{(l), k}=\sum_{g \in G} \overline{\varrho_{i j}^{(l)}(g)} e_{g}^{k}[1$, Theorem 6].

(2) $A \bar{\varrho}_{i j}^{(l), k}=\sum_{s=1}^{n} \sum_{t \in T_{k s}} \sum_{r=1}^{d_{l}} \varrho_{r i}^{(l)}(t) \bar{\varrho}_{r j}^{(l), s}$ [1, Corollary 5].

(3) $[A]_{\mathscr{B}}=\operatorname{diag}\left(I_{d_{1}} \otimes A_{1}, I_{d_{2}} \otimes A_{2}, \ldots, I_{d_{m}} \otimes A_{m}\right)$, where $A_{l}=\left[A_{i j}^{(l)}\right]$ and $A_{i j}^{(l)}=\sum_{t \in T_{j i}} \varrho^{(l)}(t)[1$, Theorem 6].

Now, let $D=\left[d_{(g, i)(h, j)}\right]_{g, h \in G, 1 \leq i, j \leq n}$ be the degree matrix of $\Gamma$. Then, $d_{(g, i)(g, i)}=\sum_{j=1}^{n}\left|T_{i j}\right|$ for all $g \in G$ and $1 \leq i \leq n$, and $d_{(g, i)(h, j)}=0$ whenever $g \neq h$ or $i \neq j$. We may view $D$ as a linear map $D: V \rightarrow V$ where $D e_{g}^{i}=\sum_{j=1}^{n} \sum_{h \in G} d_{(h, j)(g, i)} e_{h}^{j}$. Then, we have the following lemma:

Lemma 1 (1) $D e_{g}^{i}=\delta_{i} e_{g}^{i}$, where $\delta_{i}=\sum_{j=1}^{n}\left|T_{i j}\right|$.

(2) $D \bar{\varrho}_{i j}^{(l), k}=\delta_{k} \bar{\varrho}_{i j}^{(l), k}$.

Proof (1) immediately follows from the fact that $d_{(h, j)(g, i)}=0$ if and only if $j \neq i$ or $h \neq g$. We have

$$
\begin{aligned}
D \bar{\varrho}_{i j}^{(l), k} & =D\left(\sum_{g \in G} \overline{\varrho_{i j}^{(l)}(g)} e_{g}^{k}\right) \\
& =\sum_{g \in G} \overline{\varrho_{i j}^{(l)}(g)} D e_{g}^{k} \\
& =\sum_{g \in G} \overline{\varrho_{i j}^{(l)}(g)} \delta_{k} e_{g}^{k} \quad(\mathrm{By} \\
& =\delta_{k} \sum_{g \in G} \overline{\varrho_{i j}^{(l)}(g)} e_{g}^{k} \\
& =\delta_{k} \bar{\varrho}_{i j}^{(l), k},
\end{aligned}
$$

which proves (2).

Now, we are ready to prove the main result of the paper. Let $\alpha \in \mathbb{C}$ and $M_{\alpha}=$ $D+\alpha A$, where $D$ and $A$ are the degree and adjacency matrices of graph $\Gamma$. Note that $M_{-1}$ and $M_{+1}$ are Laplacian and signless Laplacian matrices of $\Gamma$, respectively.

Theorem 2 Let $\Gamma=\operatorname{Cay}\left(G ; T_{i j} \mid 1 \leq i, j \leq n\right)$ be an $n$-Cayley graph over a finite group $G, \operatorname{Irr}(G)=\left\{\varrho^{(1)}, \ldots, \varrho^{(m)}\right\}, d_{l}$ be the degree of $\varrho^{(l)}$, and $A_{l}=\left[A_{i j}^{(l)}\right]$ is $n d_{l} \times n d_{l}$ block matrix with $d_{l} \times d_{l}$ blocks $A_{i j}^{(l)}, 1 \leq i, j \leq n$, where $A_{i j}^{(l)}=$ $\sum_{t \in T_{j i}} \varrho^{(l)}(t)$ and $D_{l}=\operatorname{diag}\left(\delta_{1}, \ldots, \delta_{n}\right) \otimes I_{d_{l}}$, where $\delta_{i}=\sum_{j=1}^{n}\left|T_{i j}\right|$. Then, $M_{\alpha}(\Gamma)$ is similar to 
$\operatorname{diag}\left(I_{d_{1}} \otimes M_{1, \alpha}, I_{d_{2}} \otimes M_{2, \alpha}, \ldots, I_{d_{m}} \otimes M_{m, \alpha}\right)$,

where $M_{l, \alpha}=D_{l}+\alpha A_{l}$. In particular, $\chi_{M_{\alpha}(\Gamma)}(\lambda)=\prod_{l=1}^{m} \chi_{M_{l, \alpha}}(\lambda)^{d_{l}}$.

Proof By the proof of [1, Theorem 6], $V=\bigoplus_{l=1}^{m} \bigoplus_{j=1}^{d_{l}} V_{j}^{(l)}$, where $V_{j}^{(l)}$ is a vector space with basis $\mathscr{C}_{j}^{(l)}=\left\langle\bar{\varrho}_{i j}^{(l), k} \mid 1 \leq i \leq d_{l}, 1 \leq k \leq n\right\rangle$. By Lemma $1, V_{j}^{(l)}$ is $D$ invariant subspace of $V$ and $\left[\left.D\right|_{V_{j}^{(l)}}\right]_{\mathscr{C}_{j}^{(l)}}=\left[\left.D\right|_{V_{j^{\prime}}^{(l)}}\right]_{\mathscr{C}_{j^{\prime}}^{(l)}}$ for all $1 \leq j, j^{\prime} \leq d_{l}$. So by the primary decomposition theorem, $[D]_{\mathscr{B}}=\operatorname{diag}\left(I_{d_{1}} \otimes D_{1}, I_{d_{2}} \otimes D_{2}, \ldots, I_{d_{m}} \otimes D_{m}\right)$, where $D_{l}=\left[\left.D\right|_{V_{1}^{(l)}}\right]_{\mathscr{C}_{1}^{(l)}}, 1 \leq l \leq m$. By ordering

$$
\varrho_{11}^{(l), 1}, \varrho_{21}^{(l), 1}, \ldots, \varrho_{d_{l} 1}^{(l), 1}, \varrho_{11}^{(l), 2}, \ldots, \varrho_{d_{l} 1}^{(l), 2}, \ldots, \varrho_{11}^{(l), n}, \ldots, \varrho_{d_{l} 1}^{(l), n}
$$

of the generators of $\mathscr{C}_{1}^{(l)}$, by Lemma $1, D_{l}=\operatorname{diag}\left(\delta_{1}, \ldots, \delta_{n}\right) \otimes I_{d_{l}}$. Now, the result follows from Theorem 1(3).

\section{Corollary 1 Keeping the notations of Theorem 2,}

(1) the set of eigenvalues of $M_{\alpha}(\Gamma)$ is $\bigcup_{l=1}^{m} \xi_{l}$, where $\xi_{l}$ is the set of eigenvalues of $M_{l, \alpha}$. In particular, each eigenvalue of matrix $\left[a_{i j}\right]$, where $a_{i i}=\delta_{i}+\alpha\left|T_{i i}\right|$ and $a_{i j}=\alpha\left|T_{j i}\right|$, where $1 \leq i, j \leq n$ and $j \neq i$, is an eigenvalue of $M_{\alpha}(\Gamma)$,

(2) if the eigenvalue $\lambda$ occurs with multiplicity $m_{l}(\lambda)$ in $M_{l, \alpha}$, then the multiplicity of $\lambda$ in $M_{\alpha}(\Gamma)$ is $\sum_{l=1}^{m} d_{l} m_{l}(\lambda)$,

(3) $M_{\alpha}(\Gamma)$ is integral if and only if $M_{l, \alpha}$ is integral for all $l=1, \ldots, m$.

Let $\Gamma=\operatorname{Cay}\left(G ; T_{i j} \mid 1 \leq i, j \leq n\right)$. Let for all $g \in G$ and all $1 \leq i, j \leq n$, we have $g^{-1} T_{i j} g=T_{i j}$ (in other words $T_{i j}$ 's are a union of conjugacy classes of $G$ ). Then, we call $\Gamma$ is a quasiabelian $n$-Cayley graph.

Theorem 3 Let $\Gamma=\operatorname{Cay}\left(G ; T_{i j} \mid 1 \leq i, j \leq n\right)$ be a quasiabelian $n$-Cayley graph over $G, \operatorname{Irr}(G)=\left\{\varrho^{(1)}, \ldots, \varrho^{(m)}\right\}$ and $\eta_{l}$ be the corresponding character of $\varrho^{(l)}$. Put $c_{i j}^{(l)}=\frac{\sum_{t \in T_{j i}} \eta_{l}(t)}{d_{l}}$ and $B_{l}=\left[c_{i j}^{(l)}\right]_{1 \leq i, j \leq n}$. Then, $\chi_{M_{\alpha}(\Gamma)}(\lambda)=\Pi_{l=1}^{m} \chi_{N_{l, \alpha}}(\lambda)^{d_{l}^{2}}$, where $N_{l, \alpha}=\operatorname{diag}\left(\delta_{1}, \ldots, \delta_{n}\right)+\alpha B_{l}$.

Proof By Theorem 2, $\chi_{M_{\alpha}(\Gamma)}(\lambda)=\Pi_{l=1}^{m} \chi_{M_{l, \alpha}}(\lambda)^{d_{l}}$ where $M_{l, \alpha}=D_{l}+\alpha A_{l}, D_{l}=$ $\operatorname{diag}\left(\delta_{1}, \ldots, \delta_{n}\right) \otimes I_{d_{l}}, A_{l}=\left[A_{i j}^{(l)}\right]_{1 \leq i, j \leq n}$ and $A_{i j}^{(l)}=\sum_{t \in T_{j i}} \varrho^{(l)}(t)$.

Since $g^{-1} T_{j i} g=T_{j i}$, for every $g \in G$, we have 


$$
\begin{aligned}
\varrho^{(l)}(g) A_{i j}^{(l)} \varrho^{(l)}(g)^{-1} & =\sum_{t \in T_{j i}} \varrho^{(l)}(g) \varrho^{(l)}(t) \varrho^{(l)}\left(g^{-1}\right) \\
& =\sum_{t \in T_{j i}} \varrho^{(l)}\left(g t g^{-1}\right) \\
& =\sum_{t \in T_{j i}} \varrho^{(l)}(t) \\
& =A_{i j}^{(l)} .
\end{aligned}
$$

Hence,

$$
\begin{aligned}
A_{i j}^{(l)} & =\frac{1}{|G|} \sum_{g \in G} \varrho^{(l)}(g) A_{i j}^{(l)} \varrho^{(l)}(g)^{-1} \\
& =\frac{1}{d_{l}} \operatorname{tr}\left(A_{i j}^{(l)}\right) I_{d_{l}} \quad \text { (By Schur's Lemma) } \\
& =\frac{1}{d_{l}}\left(\sum_{t \in T_{j i}} \operatorname{tr}\left(\varrho^{(l)}(t)\right)\right) I_{d_{l}} \\
& =\frac{1}{d_{l}}\left(\sum_{t \in T_{j i}} \eta_{l}(t)\right) I_{d_{l}}
\end{aligned}
$$

Then, $A_{i j}^{(l)}=c_{i j}^{(l)} I_{d_{l}}$ and $A_{l}=\left[c_{i j}^{(l)} I_{d_{l}}\right]_{1 \leq i, j \leq n}=B_{l} \otimes I_{d_{l}}$. Hence, by Theorem 2, $\chi_{M_{\alpha}(\Gamma)}(\lambda)=\Pi_{l=1}^{m} \chi_{N_{l, \alpha}}(\lambda)^{d_{l}^{2}}$, where $N_{l, \alpha}=\operatorname{diag}\left(\delta_{1}, \ldots, \delta_{n}\right)+\alpha B_{l}$.

Example 1 Let $n$ be an odd integer, $\Gamma=\operatorname{SC}(G ; R, S, T)$, where $G=\langle a, b| a^{n}=$ $\left.b^{2}=(a b)^{2}=1\right\rangle \cong D_{2 n}$ is a dihedral group of order $2 n, R=\left\{a^{k}, a^{n-k}\right\}$ for some $1 \leq k \leq(n-1) / 2, S=\left\{b a^{j} \mid 0 \leq j \leq n-1\right\}$ and $T=\{1\}$. Then,

(1) Laplacian eigenvalues of $\Gamma$ are $2,0, n+1 \pm \sqrt{n^{2}+1}$ with multiplicity 1 and

$$
\frac{n+4-2 \cos \frac{2 k m \pi}{n} \pm \sqrt{\left(n-2+2 \cos \frac{2 k m \pi}{n}\right)^{2}+4}}{2}, \quad 1 \leq m \leq \frac{n-1}{2}
$$

with multiplicity 4.

(2) Signless Laplacian eigenvalues of $\Gamma$ are $n+3 \pm \sqrt{(n-2)^{2}+1}, 3 \pm \sqrt{5}$ with multiplicity 1 and

$$
\frac{n+4+2 \cos \frac{2 k m \pi}{n} \pm \sqrt{\left(n-2-2 \cos \frac{2 k m \pi}{n}\right)^{2}+4}}{2}, \quad 1 \leq m \leq \frac{n-1}{2}
$$

with multiplicity 4. 
Proof By [15, p. 182], $R, S$ and $T$ are conjugacy classes of $G$, and irreducible characters of $G$ are

$$
\begin{aligned}
& \mu_{1}: G \rightarrow \mathbb{C} ; \quad b^{i} a^{j} \mapsto 1, \\
& \mu_{2}: G \rightarrow \mathbb{C} ; \quad b^{i} a^{j} \mapsto(-1)^{i}, \\
& \theta_{m}: G \rightarrow \mathbb{C} ; \quad 1 \mapsto 2, a^{j} \mapsto \omega^{j m}+\omega^{-j m}, b a^{j} \mapsto 0, \quad 1 \leq m \leq(n-1) / 2,
\end{aligned}
$$

where $\omega=\exp (2 \pi i / n)$. Putting $\alpha=-1$ and $\alpha=1$ in Theorem 3, by an easy calculation, one can get the Laplacian and signless Laplacian eigenvalues of $\Gamma$, respectively.

Since every semi-Cayley graph over an abelian group is a quasiabelian semi-Cayley graph and all character degrees of abelian groups are 1, one can get the main result of [12], see [12, Theorem 1], by putting $\alpha=1,-1$ in the following corollary.

Corollary 2 Let $\Gamma=\operatorname{SC}(G ; R, S, T)$ be a quasiabelian semi-Cayley graph a group $G$ with irreducible characters $\mu_{i}, i=1, \ldots,|G|$ and $d_{i}$ be the degree of $\mu_{i}$. Then, eigenvalues of $M_{\alpha}(\Gamma)$ are

$$
\frac{a_{l} \pm \sqrt{b_{l}+4 \alpha^{2} c_{l}^{2}}}{2}
$$

where $a_{l}=|R|+|S|+2|T|+\alpha\left(\frac{\mu_{l}(R)+\mu_{l}(S)}{d_{l}}\right), b_{l}=|R|-|S|+\alpha\left(\frac{\mu_{l}(R)-\mu_{l}(S)}{d_{l}}\right)$ and $c_{l}=\frac{\left|\mu_{l}(T)\right|}{d_{l}^{2}}$.

The $n$-sunlet graph on $2 n$ vertices is obtained by attaching $n$ pendant edges to the cycle $C_{n}$.

Corollary 3 Let $\Gamma$ be an $n$-sunlet graph. Then, $M_{\alpha}(\Gamma)$ has eigenvalues

$$
2+\alpha \cos \frac{2 \pi l}{n} \pm \sqrt{\left(1+\alpha \cos \frac{2 \pi l}{n}\right)^{2}+\alpha^{2}}
$$

where $l=0, \ldots, n-1$.

Proof It is easy to see that $\Gamma=\operatorname{SC}(G, R, S, T)$, where $G=\langle a\rangle \cong \mathbb{Z}_{n}, R=\left\{a, a^{-1}\right\}$, $S=\emptyset$ and $T=\{1\}$. On the other hand, irreducible characters of $G$ are $\mu_{0}, \ldots, \mu_{n-1}$, where $\mu_{l}\left(a^{k}\right)=\cos \frac{2 \pi k l}{n}+i \sin \frac{2 \pi k l}{n}$. Now, the result follows from Corollary 2 .

Let $\Gamma=\operatorname{Cay}\left(G ; T_{i j} \mid 1 \leq i, j \leq 3\right)$ be a 3-Cayley graph. Then, $T_{i j}^{-1}=T_{j i}$ and $1 \notin T_{i i}$. We denote this graph by $\mathrm{TC}\left(G ; T_{11}, T_{22}, T_{33}, T_{12}, T_{13}, T_{23}\right)$, where $T_{i j}$ 's are the same as defined in $\Gamma$.

Corollary 4 Let $\Gamma=\mathrm{TC}\left(G ; T_{11}, T_{22}, T_{33}, T_{12}, T_{13}, T_{23}\right)$, where $G$ is a finite abelian group with irreducible characters $\mu_{1}, \ldots, \mu_{|G|}$. Then, 
(1) Laplacian eigenvalues of $\Gamma$ are the roots of $\lambda^{3}-a_{l} \lambda^{2}+b_{l} \lambda-c_{l}=0,1 \leq l \leq|G|$, where

$$
\begin{aligned}
a_{l}= & -\sum_{i=1}^{3} \mu_{l}\left(T_{i i}\right)+\sum_{i=1}^{3} \delta_{i}, \\
b_{l}= & \sum_{i, j=1, i<j}^{3}\left[\mu_{l}\left(T_{i i}\right) \mu_{l}\left(T_{j j}\right)-\left|\mu_{l}\left(T_{i j}\right)\right|^{2}\right] \\
& +\sum_{i, j=1, i<j}^{3} \delta_{i} \delta_{j}-\sum_{i, j=1, i \neq j}^{3} \delta_{i} \mu_{l}\left(T_{j j}\right), \\
c_{l}= & -\left[\mu_{l}\left(T_{11}\right) \mu_{l}\left(T_{22}\right) \mu_{l}\left(T_{33}\right)-\mu_{l}\left(T_{11}\right)\left|\mu_{l}\left(T_{23}\right)\right|^{2}-\mu_{l}\left(T_{33}\right) \mid \mu_{l}\left(T_{12}\right)^{2}\right. \\
& \left.-\mu_{l}\left(T_{22}\right)\left|\mu_{l}\left(T_{13}\right)\right|^{2}+2 \operatorname{Re}\left(\overline{\mu_{l}\left(T_{13}\right)} \mu_{l}\left(T_{12}\right) \mu_{l}\left(T_{23}\right)\right)\right]+\delta_{1} \delta_{2} \delta_{3} \\
& -\sum_{i, j, k=1, i<j, k \neq i, j}^{3} \delta_{i} \delta_{j} \mu_{l}\left(T_{k k}\right)+\sum_{i, j, k=1, i \neq j \neq k \neq i} \mu_{l}\left(T_{j j}\right) \mu_{l}\left(T_{k k}\right) \\
& -\sum_{i, j, k=1, i \neq j<k \neq i}^{3} \delta_{i}\left|\mu_{l}\left(T_{j k}\right)\right|^{2},{ }^{3}
\end{aligned}
$$

(2) Signless Laplacian eigenvalues of $\Gamma$ are the roots of $\lambda^{3}-a_{l}^{\prime} \lambda^{2}+b_{l}^{\prime} \lambda-c_{l}^{\prime}=$ $0,1 \leq l \leq|G|$, where

$$
\begin{aligned}
& a_{l}^{\prime}=\sum_{i=1}^{3} \mu_{l}\left(T_{i i}\right)+\sum_{i=1}^{3} \delta_{i} \\
& b_{l}^{\prime}=\sum_{i, j=1, i<j}^{3}\left[\mu_{l}\left(T_{i i}\right) \mu_{l}\left(T_{j j}\right)-\left|\mu_{l}\left(T_{i j}\right)\right|^{2}\right]+\sum_{i, j=1, i<j}^{3} \delta_{i} \delta_{j} \\
& +\sum_{i, j=1, i \neq j}^{3} \delta_{i} \mu_{l}\left(T_{j j}\right) \\
& c_{l}^{\prime}=\mu_{l}\left(T_{11}\right) \mu_{l}\left(T_{22}\right) \chi_{l}\left(T_{33}\right)-\chi_{l}\left(T_{11}\right)\left|\chi_{l}\left(T_{23}\right)\right|^{2} \\
& -\chi_{l}\left(T_{33}\right)\left|\chi_{l}\left(T_{12}\right)\right|^{2}-\chi_{l}\left(T_{22}\right)\left|\chi_{l}\left(T_{13}\right)\right|^{2} \\
& +2 \operatorname{Re}\left(\overline{\chi_{l}\left(T_{13}\right)} \chi_{l}\left(T_{12}\right) \chi_{l}\left(T_{23}\right)\right)+\delta_{1} \delta_{2} \delta_{3}+\sum_{i, j, k=1, i<j, k \neq i, j}^{3} \delta_{i} \delta_{j} \mu_{l}\left(T_{k k}\right) \\
& +\sum_{i, j, k=1, i \neq j \neq k \neq i}^{3} \delta_{i} \mu_{l}\left(T_{j j}\right) \mu_{l}\left(T_{k k}\right)-\sum_{i, j, k=1, i \neq j<k \neq i}^{3} \delta_{i}\left|\mu_{l}\left(T_{j k}\right)\right|^{2},
\end{aligned}
$$

where $\delta_{1}=\left|T_{11}\right|+\left|T_{12}\right|+\left|T_{13}\right|, \delta_{2}=\left|T_{12}\right|+\left|T_{22}\right|+\left|T_{23}\right|$ and $\delta_{3}=\left|T_{13}\right|+\left|T_{23}\right|+\left|T_{33}\right|$. 
Proof Since the eigenvalues of a $3 \times 3$ matrix $X$ are the roots of $\lambda^{3}-\operatorname{tr}(X) \lambda^{2}+$ $\left(\operatorname{tr}\left(X^{2}\right)-\operatorname{tr}(X)^{2}\right) \lambda-\operatorname{det}(X)=0$, (1) and (2) are direct consequences of Theorem 3 .

The adjacency eigenvalues of the Tutte's 8-cage graph are $(-3)^{[1]},(-2)^{[9]}, 0^{[10]}$, $2^{[9]}, 3^{[1]}$, see [10]. Hence, since it is a bipartite 3-regular graph, its Laplacian and signless Laplacian eigenvalues are $(6)^{[1]},(5)^{[9]}, 3^{[10]}, 1^{[9]}, 0^{[1]}$. We recompute this as an example of Corollary 4 as follows:

Example 2 Let $\Gamma$ be the Tutte's 8-cage graph. Then, $\Gamma=\operatorname{TC}\left(G ; T_{11}, T_{22}, T_{33}\right.$, $\left.T_{12}, T_{13}, T_{23}\right)$ where $G=\langle a\rangle \cong \mathbb{Z}_{10}, T_{11}=\left\{a, a^{9}\right\}, T_{22}=\left\{a^{5}\right\}, T_{33}=\left\{a^{3}, a^{7}\right\}$, $T_{12}=\{1\}, T_{13}=\varnothing$ and $T_{23}=\{1\}$. Since the irreducible characters of $G$ are $\mu_{1}, \ldots, \mu_{10}$, where $\mu_{l}\left(a^{j}\right)=\omega^{(l-1) j}$ and $\omega=\exp (\pi i / 5)$, using Corollary 4 , the Laplacian characteristic polynomial of $\Gamma$ is the product of polynomials $\lambda^{3}-4 \lambda^{2}+3 \lambda=$ $\lambda(\lambda-1)(\lambda-3),\left(\lambda^{3}-9 \lambda^{2}+23 \lambda-15\right)^{8}=(\lambda-1)^{8}(\lambda-3)^{8}(\lambda-5)^{8}$ and $\lambda^{3}-14 \lambda^{2}+63 \lambda-90=(\lambda-3)(\lambda-5)(\lambda-6)$. Hence, the Laplacian eigenvalues of $\Gamma$ are $(6)^{[1]},(5)^{[9]}, 3^{[10]}, 1^{[9]}, 0^{[1]}$.

\section{Some constructions of integral graphs from integral Cayley graphs}

In this section, we assume that $G$ is a group with $\operatorname{Irr}(G)=\left\{\varrho^{(1)}, \ldots, \varrho^{(m)}\right\}$ and $\varrho^{(1)}$ is the trivial representation of $G$. Also, if $\Gamma=\operatorname{Cay}\left(G ; T_{i j} \mid 1 \leq i, j \leq n\right)$, then $\delta_{i}=\sum_{j=1}^{n}\left|T_{i j}\right|, 1 \leq i \leq n$. We know that $|S|$ is an eigenvalue of $\operatorname{Cay}(G, S)$. We call this eigenvalue trivial eigenvalue of $\operatorname{Cay}(G, S)$. Also, the remaining eigenvalues of Cay $(G, S)$ are eigenvalues of $\varrho^{(l)}(S)$ with multiplicity $d_{l}$, where $l=2, \ldots, m$, see [1, Corollary 7]. In what follows, using Theorem 2, we construct some Laplacian and signless Laplacian integral graphs of order $n|G|$, where $n \geq 2$.

Corollary 5 Let $\Gamma=\operatorname{Cay}\left(G ; T_{i j} \mid 1 \leq i, j \leq n\right)$, where $T_{i j}=$ G for all $i \neq j$. Then, Laplacian eigenvalues of $\Gamma$ are 0 with multiplicity $1, n|G|$ with multiplicity $n-1$ and $\left|T_{i i}\right|+(n-1)|G|-\theta$, where $\theta$ is a non-trivial eigenvalue of $\operatorname{Cay}\left(G, T_{i i}\right)$, $i=1, \ldots, n$. In particular, if $\operatorname{Cay}\left(G, T_{i i}\right)$ is integral for all $i=1, \ldots, n$, then $\Gamma$ is Laplacian integral.

Proof Since for all $l=2, \ldots, m$, we have $\sum_{g \in G} \varrho^{(l)}(g)=0_{d_{l}}$, where $d_{l}$ is the degree of $\varrho^{(l)}$ and $0_{d_{l}}$ is the $d_{l} \times d_{l}$ zero matrix, by Theorem 2 the Laplacian eigenvalues of $\Gamma$ are the eigenvalues of $n|G| I_{n}-|G| J_{n}$, where $J_{n}$ is the $n \times n$ all ones matrix, and eigenvalues of $\operatorname{diag}\left(\delta_{1} I_{d_{l}}-\varrho^{(l)}\left(T_{11}\right), \delta_{2} I_{d_{l}}-\varrho^{(l)}\left(T_{22}\right), \ldots, \delta_{n} I_{d_{l}}-\varrho^{(l)}\left(T_{n n}\right)\right)$ with multiplicity $d_{l}$, where $l=2, \ldots, m$. On the other hand, $\delta_{i}=\left|T_{i i}\right|+(n-1)|G|$ and $J_{n}$ has eigenvalues $n$ with multiplicity 1 and 0 with multiplicity $n-1$. This leads us to the result.

Corollary 6 Let $\Gamma=\operatorname{SC}(G ; R, S, G)$. Then, signless eigenvalues of $\Gamma$ are

$$
|G|+|R|+|S| \pm \sqrt{(|R|-|S|)^{2}+|G|^{2}}
$$


$|R|+|G|+\theta$ and $|S|+|G|+\gamma$, where $\theta$ and $\gamma$ are non-trivial eigenvalues of $\operatorname{Cay}(G, R)$ and $\operatorname{Cay}(G, S)$, respectively. In particular, if $\operatorname{Cay}(G, R)$ and $\operatorname{Cay}(G, S)$ are integral and $|R|=|S|$, then $\Gamma$ has integral signless Laplacian eigenvalues.

Proof Since for all $l=2, \ldots, m$, we have $\sum_{g \in G} \varrho^{(l)}(g)=0_{d_{l}}$, where $d_{l}$ is the degree of $\varrho^{(l)}$ and $0_{d_{l}}$ is the $d_{l} \times d_{l}$ zero matrix, by Theorem 2 signless Laplacian eigenvalues $\Gamma$ are the eigenvalues of

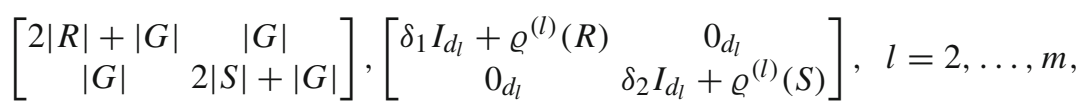

where $\delta_{1}=|R|+|G|$ and $\delta_{2}=|S|+|G|$. The eigenvalues of the right-hand side matrix are $\lambda$ and $\mu$, where $\lambda$ and $\mu$ are eigenvalues of $\delta_{1} I_{d_{l}}+\varrho^{(l)}(R)$ and $\delta_{2} I_{d_{l}}+\varrho^{(l)}(S)$, respectively. These leads us to the result.

Corollary 7 Let $\Gamma=\operatorname{Cay}\left(G ; T_{i j} \mid 1 \leq i, j \leq n\right)$, where $T_{i i}=R$ and $T_{i j}=\{1\}$ for all $1 \leq i \leq j \leq n$ and $j \neq i$. Then, eigenvalues of $M_{\alpha}(\Gamma)$ are $|R|+n-1+\alpha(n+\lambda-1)$ with multiplicity 1 and $|R|+n-1+\alpha(\lambda-1)$ with multiplicity $n-1$, where $\lambda$ is an eigenvalue of $\operatorname{Cay}(G, R)$. In particular, if $\operatorname{Cay}(G, R)$ is integral, then $\Gamma$ is Laplacian and signless Laplacian integral.

Proof By Theorem 2, eigenvalues of $M_{\alpha}(\Gamma)$ are eigenvalues of

$$
(|R|+n-1) I_{n d_{l}}+\alpha A_{l}
$$

with multiplicity $d_{l}, l=1, \ldots, m$, where $A_{l}=\left[a_{i j}\right]_{1 \leq i, j \leq n}, a_{i i}=\varrho^{(l)}(R)$ and $a_{i j}=I_{d_{l}}$ for $i \neq j$. Let $\lambda$ be an eigenvalue of $\varrho^{(l)}(R)$ with eigenvector $v$. Then, $u=\left(u_{1}, \ldots, u_{n}\right)$ is an eigenvector of $A_{l}$ with eigenvalue $n+\lambda-1$ whenever $u_{1}=$ $\ldots=u_{n}=v$. Also, the $n-1$ independent vectors

$$
(-(n-1) v, v, \ldots, v),(v,-(n-1) v, v, \ldots, v), \ldots,(v, \ldots, v,-(n-1) v, v)
$$

are eigenvectors of $A_{l}$ corresponding to $\lambda-1$. This shows that eigenvalues of $A_{l}$ are $n+\lambda-1$ with multiplicity 1 and $\lambda-1$ with multiplicity $n-1$, where $\lambda$ is an eigenvalue of $\varrho^{(l)}(R)$. Hence, eigenvalues of $M_{\alpha}(\Gamma)$ are $|R|+n-1+\alpha(n+\lambda-1)$ with multiplicity 1 and $|R|+n-1+\alpha(\lambda-1)$ with multiplicity $n-1$, where $\lambda$ is an eigenvalue of $\operatorname{Cay}(G, R)$.

Corollary 8 Let $\Gamma=\operatorname{Cay}\left(G ; T_{i j} \mid 1 \leq i, j \leq n\right)$ where $T_{i j}=R$ for all $1 \leq$ $i, j \leq n$. Then, eigenvalues of $M_{\alpha}(\Gamma)$ are $n(|R|+\alpha \theta)$ with multiplicity 1 , where $\theta$ is an eigenvalue of $\operatorname{Cay}(G, R)$ and $n|R|$ with multiplicity $(n-1)|G|$. In particular, if $\operatorname{Cay}(G, R)$ is integral, then $\Gamma$ is Laplacian and signless Laplacian integral. 
Proof By Theorem 2, eigenvalues of $M_{\alpha}(\Gamma)$ are the eigenvalues of $n|R| I_{n d_{l}}+\alpha J_{n} \otimes$ $\varrho^{(l)}(X)$ with multiplicity $d_{l}$, where $l=1, \ldots, m$. Eigenvalues of $J_{n}$ are 0 with multiplicity $n-1$ and $n$ with multiplicity 1 , which leads us to the result.

\section{References}

1. Arezoomand, M., Taeri, B.: On the characteristic polynomial of $n$-Cayley digraphs. Electron. J. Combin. 20(3), P57 (2013)

2. Arezoomand, M., Taeri, B.: Isomorphisms of finite semi-Cayley graphs. Acta Math. Sin. (Engl. Ser.) 31(4), 715-730 (2015)

3. Arezoomand, M., Taeri, B.: A classification of finite groups with integral bi-Cayley graphs. Trans. Comb. 4(4), 55-61 (2015)

4. Arezoomand, M., Taeri, B.: Finite groups admitting a connected cubic bi-Cayley graph. Alg. Struc. Appl. 5(2), 35-43 (2018)

5. Biggs, N.: Algebraic Graph Theory. Cambridge University Press, Cambridge (1974)

6. Cvetković, D., Simić, S.K.: Towards a spectral theory of graphs based on the signless Laplacian, II. Linear Algebra Appl. 432(9), 2257-2272 (2010)

7. Du, S.F., Marušič, D.: An infinite family of biprimitive semisymmetric graphs. J. Graph Theory 32(3), 217-228 (1999)

8. Du, S.F., Marušič, D.: Biprimitive graphs of smallest order. J. Algebraic Combin. 9(2), 151-156 (1999)

9. Fallat, S., Kirkland, S., Molitierno, J., Neumann, M.: On graphs whose Laplacian matrices have distinct integer eigenvalues. J. Graph Theory 50(2), 162-174 (2005)

10. Frucht, R.: A canonical representation of trivalent Hamiltonian graphs. J. Graph Theory 1(1), 45-60 (1976)

11. Gao, X., Luo, Y.: The spectrum of semi-Cayley graphs over abelian groups. Linear Algebra Appl. 432(11), 2974-2983 (2010)

12. Gao, X., Lü, H., Hao, Y.: The Laplacian and signless Laplacian spectrum of semi-Cayley graphs over abelian groups. J. Appl. Math. Comput. 51(1), 383-395 (2016)

13. Hammer, P., Kelmans, A.: Laplacian spectra and spanning trees of threshold graphs. Discrete Appl. Math. 65(1-3), 255-273 (1996)

14. Huang, X., Huang, Q., Wen, F.: On the Laplacian integral tricyclic graphs. Linear Multilinear Algebra 63(7), 1356-1371 (2014)

15. James, G., Liebeck, M.: Representations and Characters of Groups, 2nd edn. Cambridge University Press, Cambridge (2003)

16. Kirkland, S.: Completion of Laplacian integral graphs via edge addition. Discrete Math. 295(1-3), 75-90 (2005)

17. Kirkland, S.: Constructably Laplacian integral graphs. Linear Algebra Appl. 423(1), 3-21 (2007)

18. Kovács, I., Malnič, I., Marušič, D., Miklavič, Š.: One-matching bi-Cayley graphs over abelian groups. European J. Combin. 30(2), 602-616 (2009)

19. Kutnar, K., Marušič, D., Miklavič, Š., Šparl, P.: Strongly regular tri-Cayley graphs. European J. Combin. 30(4), 822-832 (2009)

20. Leung, K.H., Ma, S.L.: Partial difference triples. J. Algebraic Combin. 2(4), 397-409 (1993)

21. Lu, Z.P., Wang, C.Q., Xu, M.Y.: Semisymmetric cubic graphs constructed from bi-Cayley graphs of $A_{n}$. Ars Combin. 80, 177-187 (2006)

22. Martinez, L.: Strongly regular $m$-Cayley circulant graphs and digraphs. Ars Math. Contemp. 8(1), 195-213 (2015)

23. Marušič, D.: Strongly regular bicirculants and tricirculants. Ars Combin. 25C, 11-15 (1988)

24. Merris, R.: Degree maximal graphs are Laplacian integral. Linear Algebra Appl. 199(suppl 1), 381-389 (1994)

25. Merris, R.: Large families of Laplacian isospectral graphs. Linear Multilinear Algebra 43(1-3), 201205 (1997)

26. Mohar, B.: The Laplacian spectrum of graphs, graph theory, combinatorics and applications. In: Proceedings of the Sixth Quadrennial International Conference on the Theory and Applications of Graphs, Western Michigan University, vol. 2, pp. 871-898 (1991) 
27. Resmi, M.J.D., Jungnickel, D.: Strongly regular semi-Cayley graphs. J. Algebraic Combin. 1(2), 171195 (1992)

28. Simič, S.K., Stanič, Z.: On $Q$-integral $(3, s)$-semiregular bipartite graphs. Appl. Anal. Discrete Math. 4(1), 167-174 (2010)

29. Stanič, Z.: Some results on $Q$-integral graphs. Ars Combin. 90, 321-335 (2009)

30. Zhao, G., Wang, L., Li, K.: $Q$-integral complete $r$-partite graphs. Linear Algebra Appl. 438(3), 10671077 (2013)

Publisher's Note Springer Nature remains neutral with regard to jurisdictional claims in published maps and institutional affiliations. 